Journal of Applied Sciences 5 (1): 80-84, 2005

ISSN 1607-8926

(C) 2005 Asian Network for Scientific Information

\title{
Three-phase Single Switch Power Factor Correction Circuit with Harmonic Reduction
}

\author{
S.M. Bashi, N. Mariun, S.B. Noor and H.S. Athab \\ Department of Electrical and Electronic Engineering, Faculty of Engineering, \\ University Putra Malaysia, 43400 UPM Serdang, Selangor, Malaysia
}

\begin{abstract}
A harmonic injection technique, which reduces the line frequency harmonics of the single switch three-phase boost rectifier, has been implemented. In this method, a periodic voltage is injected in the control circuit to vary the duty cycle of the rectifier switch within a line cycle so that the fifth-order harmonic of the input current is reduced to meet the total harmonic distortion (THD) requirement. Since the injected voltage signal, which is proportional to the inverted ac component of the rectified three-phase line-to-line input voltages is employed; the injected duty cycle variations are naturally synchronized with the three-phase line-toneutral input voltages.
\end{abstract}

Key words: Boost converter, discontinuous conduction mode, harmonic injection, power factor correction, three-phase rectifier

\section{INTRODUCTION}

In most of the power electronics applications, diode rectifiers are commonly used in the front end of a power converter as an interface with the electric utility. The rectifiers are nonlinear in nature and, consequently, generate harmonic currents into the ac power source. The nonlinear operation of the diode rectifiers causes highly distorted input current. The non-sinusoidal shape of the input current drawn by the rectifiers causes a number of problems in the sensitive electronic equipment and in the power distribution network. The distorted input current flowing through the system produces distorted voltages at the input of the common coupling. Thus, the increased harmonic currents result in increasing volt-ampere ratings of the utility equipment, such as generators, transmission lines and transformers. In addition to the inefficient use of electric energy, the discontinuous conduction of the bridge rectifier results in a high total harmonic distortion (THD) in the input lines and can lead to malfunctioning of the sensitive electronic equipment. The recommended practice, IEEE-519 and IEC 1000-3 have evolved to maintain utility power quality at acceptable levels.

In order to meet IEEE-519 and IEC 1000-3, a cost effective and economical solution to mitigate harmonics generated by power electronic equipment is currently of high interest. One approach is to use three single-phase power factor corrected rectifiers in cascade ${ }^{[1]}$. The main advantage of this configuration is that a well-known single-phase power factor correction ( $\mathrm{PFC}$ ) technique can be used in three-phase applications. However, this approach suffers from several disadvantages, which include cascading three single-phase $\mathrm{PFC}$ circuits requires the use of additional diodes, increased component count and complicated input synchronization logic. Therefore, it is clear that lower cost three-phase PFC circuits are required for high power processing.

Amongst the three-phase ac-to-dc rectifiers, boost type topologies are frequently used because of continuous input currents and high output voltages. Basically, two topologies are most popular: a six-switches full-bridge boost rectifier and a single switch boost rectifier. The first one uses six switches to achieve sinusoidal input current control and to share the output power, resulting in features, which include continuous input current, excellent power factor and low switch current rating ${ }^{[2-4]}$. However, this circuit is very complicated in power stage and control, making it too expensive for medium power level $(5-10 \mathrm{kw})$ applications. The second one uses six diodes and one switch to control input currents and output power as depicted in Fig. $1^{[5,6]}$. Since these rectifiers have a single switch and perform input current wave-shaping naturally, without a need for a complex control circuitry, they are very suitable for the low cost power three-phase ac-dc applications. In addition, they can achieve extremely high efficiencies because the reverse-recovery-related losses of the boost diode are eliminated. However, if a discontinuous

Corresponding Author: Dr. Senan Mahmod Bashi, Department of Electrical and Electronic Engineering, Faculty of Engineering, University Putra Malaysia, 43400 UPM Serdang, Selangor, Malaysia E-mail: senan@eng.upm.edu.my 


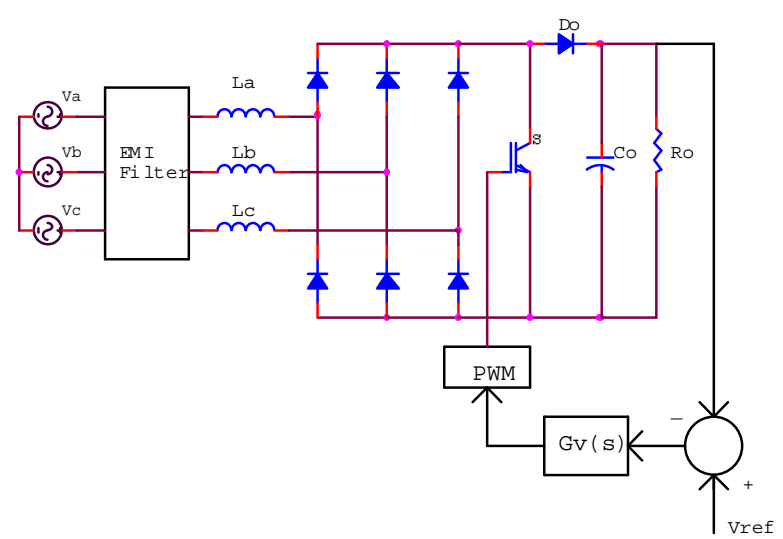

Fig. 1: Boost-star three-phase single switch PFC

conduction mode (DCM) PWM boost rectifier is implemented with the conventional constant frequency low bandwidth output voltage feed back control, which keeps the duty cycle of the switch constant during a rectified line period, the rectifier input current exhibits a relatively large fifth-order harmonic. As previous studies have shown, the distortion level depends on the ratio of the output voltage to the input line voltage. If for example, the output voltage is $720 \mathrm{~V}$ and the input phase voltage $230 \mathrm{~V}$, the maximum power of the converter must be limited to about $4.5 \mathrm{~kW}$ in order to keep input current harmonics under the limits set by IEC 1000-3. Although increasing the output voltage would permit higher maximal power, it is not practical considering the voltage handling capability of commercially available high frequency power semiconductor devices (MOSFET's and IGBT's).

To alleviate this problem to some extent, different modulation techniques have been proposed to reduce the harmonic distortion of the input currents without increasing the output voltage beyond practical levels. The first approach proposed to improve the harmonic distortion of the input currents involved operating the single switch boost rectifier in the critical mode ${ }^{[7-8]}$. To do this, the power switch must be turned on at the instant at which the boost diode current reaches zero. As a result, the switching frequency becomes variable and the effective duty cycle modulation over the line cycle results in reduced THD of the input currents. The drawback of operating the discontinuous conduction mode (DCM) boost rectifier in the critical mode is the wide range switching frequency variation that depends upon both load and input voltage limits. Another approach for improving the THD of the input currents involves controlling to a constant level the average current in the boost diode ${ }^{[4]}$. In order to keep the average current constant through the boost diode, the duty cycle must be modulated over the line cycle, resulting in an improved input current waveform. The drawback of this method is the extra current sensor required to control the average boost diode current.

A simple technique that can be used to reduce the harmonic distortion of the input current is the so-called harmonic injection method ${ }^{[9-11]}$. The principles for achieving optimal harmonic injection are described in $\operatorname{Sun}^{[0]}$. The injected signal modifies the duty cycle of the rectifier switch so that the fifth-order harmonic of the input current and the overall THD's are reduced to meet the IEC1000-3 requirement. However, if the phase of the injected signal is not well synchronized with the fifthorder harmonic of the input current, the expected reduction of the fifth-order harmonic and the improvement of the THD's cannot be achieved.

In this study, a low cost harmonic injection method for single switch three-phase DCM boost rectifier and its implementation are presented. To reduce the fifth-order harmonic and improve THD's of the rectifier input currents, a periodic voltage signal, which utilizes the voltage ripple of the rectifier output voltage, is injected to modify the duty cycle of the rectifier within line cycle. The injected voltage signal is proportional to the ac component of the rectified three-phase line-to-line input voltages. As a result, the injected signal is naturally synchronized with the three-phase line-to-neutral input voltages. Moreover, the closed loop feedback control of the DCM boost rectifier is not affected by the proposed open loop harmonic injection method.

Converter operation: The converter circuit is depicted in Fig. 1. The principle can be easily understood by assuming first that the rectifier operates in DCM and the switch $\mathrm{S}$ is operated at constant switching frequency $\left(\mathrm{f}_{\mathrm{s}}\right)$, with a fixed duty ratio. Since the boost rectifier is operated in DCM with constant frequency and constant duty cycle, all three-phase currents $i_{a}, i_{b}, i_{c}$ are zero at the end of a switching period immediately before boost switch $\mathrm{S}$ is turned on. Four operating stages can be identified in a switching period as shown in Fig. 2. In the first operating stage, the power switch is turned on to linearly charge the input inductors according to the phase voltage that is applied across each one. In the second operating stage, the power switch is turned off to reset the inductors, the inductor with the lowest peak current reset first. In the third operating stage, the two remaining inductor currents are reset to zero at the same rate. Once the reset interval has finished, the output load is supplied by the energy stored in the output capacitor until the next switching period restart. 


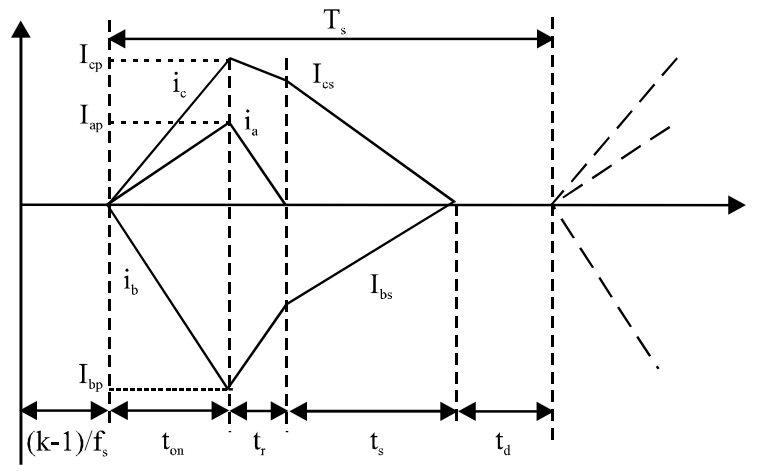

Fig. 2: (a) Input inductor currents for a switching period
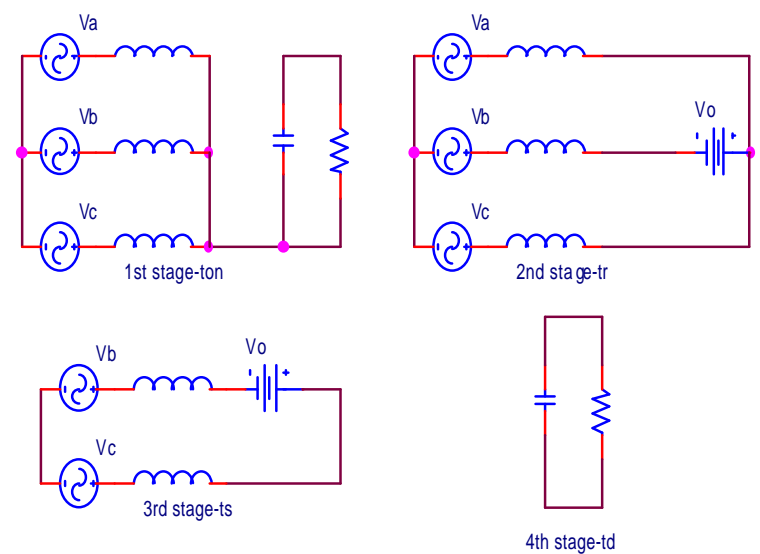

Fig. 2: (b) Operating stages of the converter

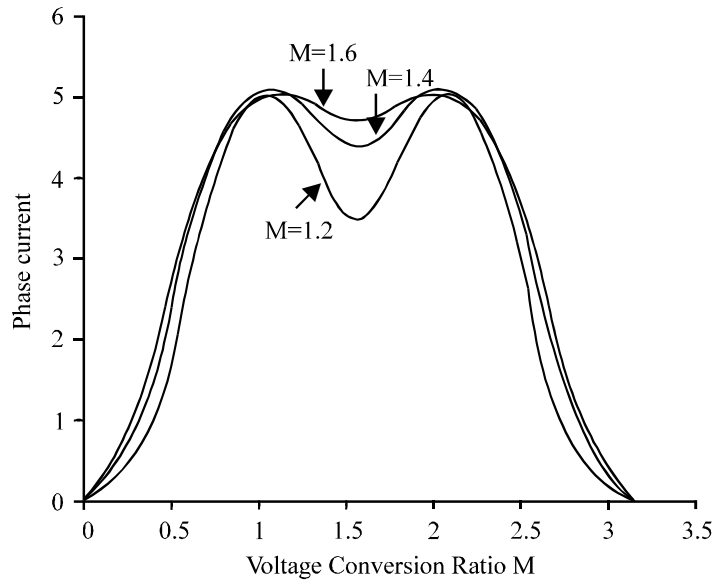

Fig. 3: Average input current for different values of $\mathrm{M}$

The average line current during a switching period of a constant frequency constant duty cycle DCM boost rectifier were derived in Simonetti et al. ${ }^{[7]}$ and Kolar et al. ${ }^{[8]}$. The derivations assume that switching frequency $\left(f_{s}\right)$ is much higher than line frequency $f_{L}$; the well-balanced and undistorted three-phase input voltages can be constant within each switching cycle. Therefore, by averaging the input current in a switching period, its higher-order harmonics are filtered out. The shape of the resulting input current for some $M$ values ( $M$ is the ratio output voltage /input peak voltage), the average value of $i_{a}$ is drawn in Fig. 3. It can be observed that the current shape is completely determined by the voltage conversion ratio $M$ : the higher the $M$, the lower the distortions.

The modulated duty cycle with harmonic injection is given by:

$$
d(t)=D[1-m \cos 6 w t .]
$$

Where, $d(t)$ is the modulated duty cycle, D is the duty cycle in the absence of the modulation and $\mathrm{m}$ is the modulation index.

\section{RESULTS}

The block diagram of the control circuit for the converter is shown in Fig. 4. A three-phase bridge rectifier followed by band-pass filter is used to generate the sixth-order harmonic and a multiplier is used to modify the amplitude of the sixth-order harmonic by the modulation index. The modulated signal is applied as the control input to a pulse width Modulator. In this control scheme, the PWM signal is obtained by monitoring the rectified input voltage and the dc output voltage only the control circuit. There is no need to monitor the current as the case of continuous mode of operation. Since the boost circuit works in discontinuous conduction mode (DCM), stability is not a problem.

The converter has been tested with the following parameters:

- Input:

$3 \times 110 \mathrm{~V} / 50 \mathrm{~Hz}$,

- Output: $250 \mathrm{~V} / 1.1 \mathrm{~kW}$

- Switching frequency:

$45 \mathrm{kHz}$

- Modulation index m:

4.6

- Control band width:

$200 \mathrm{~Hz}$

- Input inductor:

$50 \mu \mathrm{H}$

- Output capacitor: $220 \mu \mathrm{F}$

In the experimental prototype, an IGBT (IRG4PC50KD) is used as the main switch, the input bridge is constructed with MUR1560 ultra fast recovery diodes and DSEI60-10A is the output diode. Figure 5 shows the pre-filtered line current of phase $\mathrm{A}$, which contains high frequency components. It can be seen that the current always starts at zero during each switching 


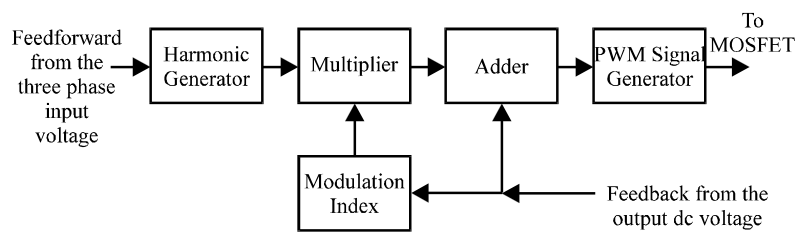

Fig. 4: Block diagram of control circuit

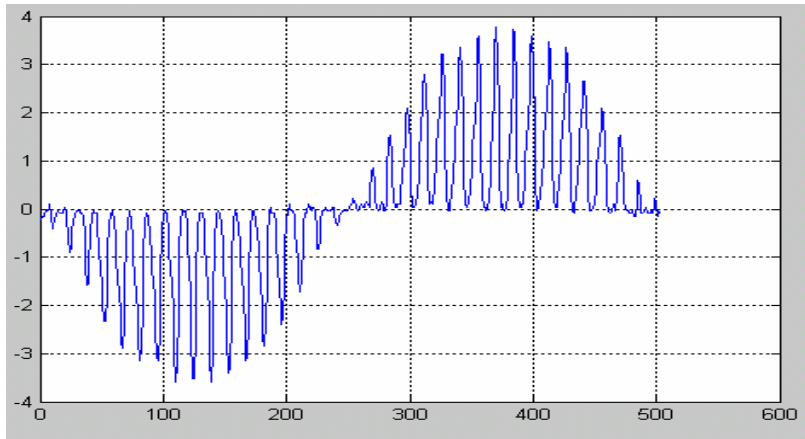

Fig. 5: Measured inductor input current

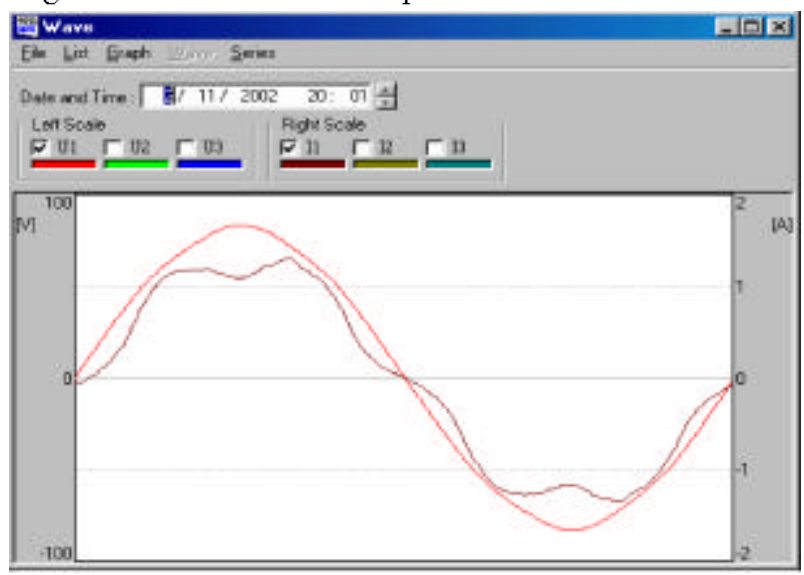

Fig. 6: Input current of phase A and its phase voltage before injection

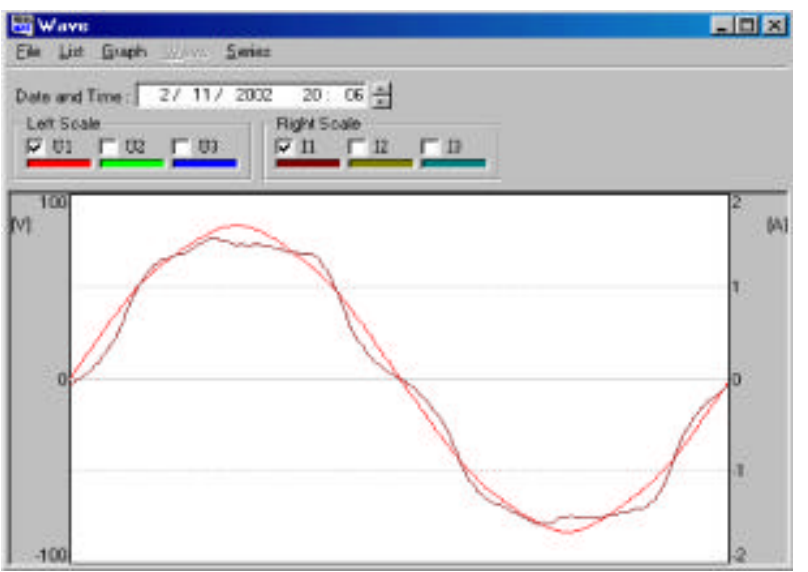

Fig. 7: Input current of phase A and its phase voltage after injection

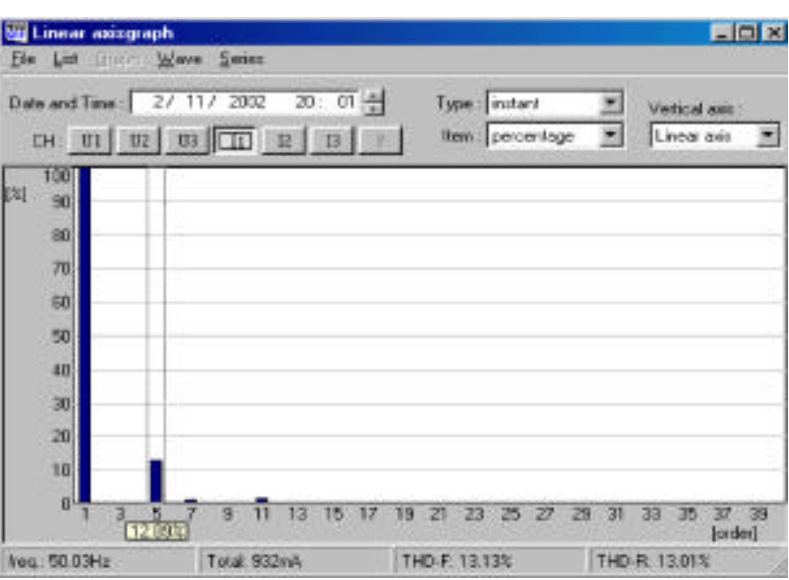

Fig. 8: Measured harmonics contents of Input current before injection

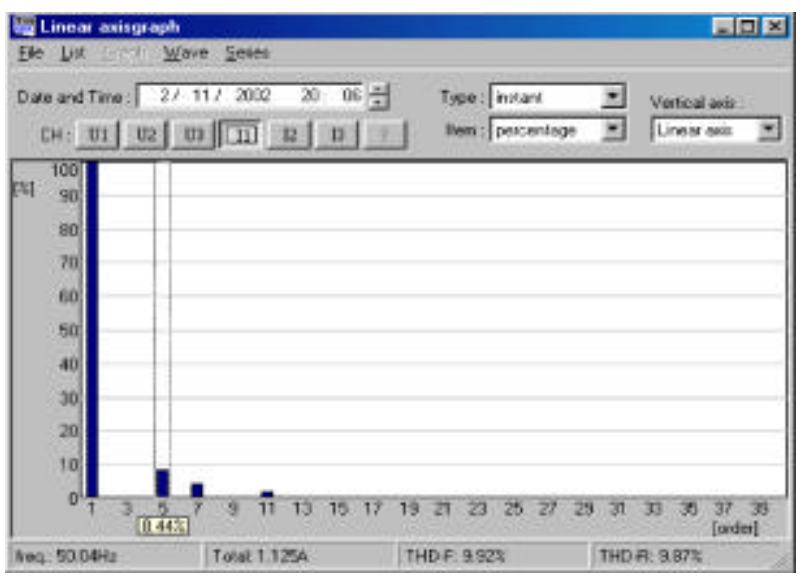

Fig. 9: Measured harmonic contents input current after injection

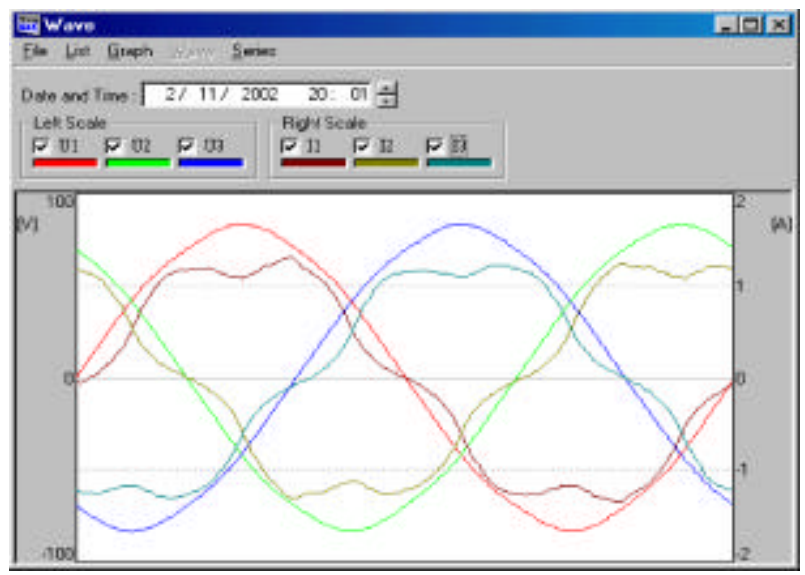

Fig. 10: Measured three-phase input current before injection 


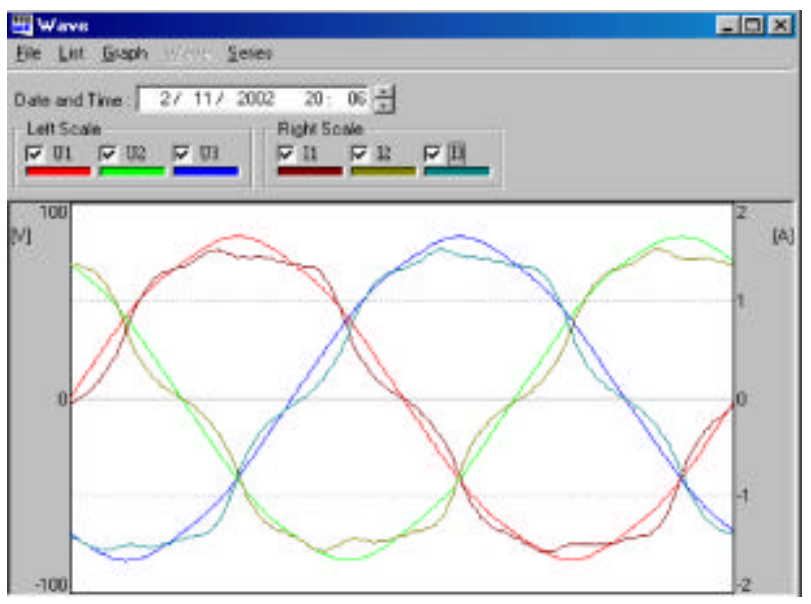

Fig. 11: Measured three-phase input currents after harmonics injection

period and its peak value takes the envelope of the phase voltage and in phase with it. Therefore, it is clear that the boost converter is well operating in discontinuous current mode (DCM) with the designed values of boost inductors. Figure 6 and 7 show the input current and its phase voltage without and with injection, respectively. Figure 8 and 9 show the harmonic spectrum of converter input current before and after injection. From the spectrum it can be seen that the fifth-order harmonic is the dominant order. By using the injection approach the fifth order harmonic is reduced the total harmonic distortion (TDH) is improved although the seventh-order is increased. Figure 10 and 11 show the three-phase current and voltages together, before and after injection.

\section{CONCLUSION}

In this study, a low cost harmonic injection method for single switch three-phase discontinuous conduction current mode boost rectifiers is implemented. In this method, a periodic voltage, which is proportional to the inverted ac component of the rectified three-phase input voltages is injected in the control circuit to vary the duty cycle of the rectifier switch within a line cycle so that the fifth-order harmonic is reduced and the THD in single switch rectifier is improved, specially for lower $M$ values. Moreover, the injected duty cycle variations are naturally synchronized with the three-phase line-to-neutral input voltages without using expensive phase-detecting and phase-looking circuits.

From the results of analysis and experimental prototype, it can be concluded that: to meet the $\mathrm{THD}<10 \%$ requirement, the rectifier voltage gain $\mathrm{M}$ can be designed down to 1.45 instead of 1.65 without injection and owing to constant switching frequency and load independent harmonic injection it is easy to design control circuit and EMI filter.

\section{REFERENCES}

1. Kocher, M.J. and R.L. Steigerwald, 1983. An AC to DC converter with high quality input waveforms. IEEE Trans. Industrial Applications., 1A-19: 586-599.

2. Jiang, Y., H. Mao, F.C. Lee and D. Boroyevich, 1994. Simple high performance three-phase boost rectifier. Conference Record IEEE PESC, pp: 1158-1163.

3. Fukuda, S. and K. Koizumi, 1995. Optimal Control of a Thee-Phase Boost Rectifier for Unity Power Factor and Reduced Harmonics. IEEE In Power Electronics and Drive System, pp: 34-39.

4. Chattopadhyay, S. and V. Ramanarayanan, 2001. Digital implementation of a line current shaping algorithm for three-phase high power factor boost rectifier without input voltage sensing. IEEE APEC, pp: 592-598.

5. Prasad, A.R., P.D. Ziogas and S. Manias, 1989. An active power factor correction technique for three phase diode rectifiers. Conference Record IEEE PESC, pp: 58-65.

6. Sedigly, M. and F.P. Dawson, 1995. Single switch three-phase power factor correction. Conference Record IEEE IPEC, pp: 293-297.

7. Simonetti, D.S.L., J. Sebastian and J. Uceda, 1993. Single-switch three-phase power factor preregulator under variable switching frequency and discontinuous input current. Conference Record IEEE PESC, pp: 657-662.

8. Kolar, J.W., H. Ertl andF.C. Zach, 1993. Space vectorbased analytical analysis of the input current distortion of a three-phase discontinuous-mode boost rectifier system. Conference Record IEEE PESC, pp: 696-703.

9. Sun, J., etc, 1996. Harmonic reduction techniques for single switched three-phase boost rectifier. Conference Record IEEE IAS, pp: 1225-1232.

10. Huang, Q. and F.C. Lee, 1996. Harmonic reduction in a single switch three-phase boost rectifier with high order harmonic injected. In IEEE PESC, 1996, 2: $1266-1271$.

11. Jang, Y. and M.M. Jovanovic, 2000. A new input-voltage feed-forward harmonic-injection with nonlinear gain control for single switch, Three-phase, DCM boost rectifier. IEEE Transactions on Power Electronics, 28: 268-277. 